Proc. Estonian Acad. Sci. Phys. Math., 2001, 50, 3, 145-154

\title{
FAST SOLVERS OF GENERALIZED AIRFOIL EQUATION OF INDEX -1
}

\author{
Gennadi VAINIKKO
}

Helsinki University of Technology, Institute of Mathematics, Otakaari 1M, 02150 Espoo, Finland; Gennadi.Vainikko@hut.fi

Received 28 May 2001

Abstract. We consider the generalized airfoil equation in the situation where the index of the problem is -1 . We periodize the problem, then discretize it by a fully discrete version of the trigonometric collocation method and apply the conjugate gradient method to solve the discretized problem. The approximate solution appears to be of optimal accuracy in a scale of Sobolev norms, and the $N$ parameters of the approximate solution can be determined by $\mathcal{O}(N \log N)$ arithmetical operations.

Key words: airfoil equations, fast solvers.

\section{THE GENERALIZED AIRFOIL EQUATION AND ITS PERIODIZATION}

Consider the generalized airfoil equation

$$
\begin{gathered}
(B v)(x):=\int_{-1}^{1}\left(\frac{1}{\pi} \frac{1}{x-y}+b_{1}(x, y) \log |x-y|+b_{2}(x, y]\right) v(y) d y=g(x), \\
-1<x<1 .
\end{gathered}
$$

We assume that the kernel functions $b_{1}$ and $b_{2}$ are smooth. It is well known (see, e.g., $\left.\left[{ }^{1-4}\right]\right)$ that $B$ represents a linear continuous Fredholm operator in different weighted spaces $L_{\sigma}^{2}(-1,1)$; the index of $B$ depends on the weight. Particularly, ind $(B)=0$ if $\sigma(x)=\sqrt{(1+x) /(1-x)}$ or $\sigma(x)=\sqrt{(1-x) /(1+x)}$, and 
ind $(B)=1$ if $\sigma(x)=\sqrt{1-x^{2}}$. Collocation solvers of Eq. (1) in these cases have been examined in $\left[{ }^{2}\right]$ and $\left[{ }^{4}\right]$, respectively. In the present paper, we put

$$
\sigma(x)=\frac{1}{\sqrt{1-x^{2}}}, \quad(u, v)_{L_{\sigma}^{2}}=\int_{-1}^{1} \sigma(x) u(x) \overline{v(x)} d x
$$

then the index of $B \in \mathcal{L}\left(L_{\sigma}^{2}(-1,1)\right)$ is -1 . We assume that the homogeneous equation $B v=0$ has in $L_{\sigma}^{2}(-1,1)$ only the trivial solution $v=0$; then the range $\mathcal{R}(B)=B L_{\sigma}^{2}(-1,1)$ is of codimension 1. Let us fix a smooth function $\psi \in L_{\sigma}^{2}(-1,1)$ outside $\mathcal{R}(B)$. For any $g \in L_{\sigma}^{2}(-1,1)$ there exists a unique pair $(\omega, v) \in \mathbb{C} \times L_{\sigma}^{2}(-1,1)$ satisfying $\omega \psi+B v=g$, and this pair can be treated as a generalized solution of (1). If $g \in \mathcal{R}(B)$, then $\omega=0$, and the generalized solution $(0, v)$ can be identified with the usual solution $v \in L_{\sigma}^{2}(-1,1)$ of (1). In the sequel we design a numerical method yielding approximations $\left(\omega_{N}, v_{N}\right)$ such that $\left|\omega_{N}-\omega\right| \rightarrow 0,\left\|v_{N}-v\right\|_{L_{\sigma}^{2}} \rightarrow 0$ with a certain velocity. Thus, the convergence $\omega_{N} \rightarrow 0$ as $N \rightarrow \infty$ indicates that $\omega=0, g \in \mathcal{R}(B)$, and (1) is solvable in $L_{\sigma}^{2}(-1,1)$ in the usual sense. An interpretation of the generalized solution $(\omega, v)$ with $\omega \neq 0$ can be given considering the flow ejection through a point of the airfoil (see $\left.\left[{ }^{5}\right]\right)$. In any case, the generalized solution $(\omega, v)$ is of interest also if $\omega \neq 0$, i.e. $g \notin \mathcal{R}(B)$. So we do not assume that $g \in \mathcal{R}(B)$.

With the cosine transformation

$$
x=x(t)=-\cos (2 \pi t)\left(0 \leq t \leq \frac{1}{2}\right), \quad y=x(s)=-\cos (2 \pi s)\left(0 \leq s \leq \frac{1}{2}\right),
$$

Eq. (1) can be reduced (see $\left[^{3}\right]$ for details) to the 1-periodic integral equation

$$
\mathcal{A} u:=A_{0} u+A_{1} u+A_{2} u=f,
$$

where

$$
\begin{aligned}
\left(A_{0} u\right)(t) & =\int_{-1 / 2}^{1 / 2} \cot \pi(t-s) u(s) d s \text { (the Hilbert transformation), } \\
\left(A_{1} u\right)(t) & =\int_{-1 / 2}^{1 / 2} a_{1}(t, s) \log |\sin \pi(t-s)| u(s) d s \\
\left(A_{2} u\right)(t) & =\int_{-1 / 2}^{1 / 2} a_{2}(t, s) u(s) d s
\end{aligned}
$$




$$
\begin{gathered}
f(t)=g(x(t)), \quad t \in \mathbb{R}, \\
a_{1}(t, s)=b_{1}(x(t), x(s)) x^{\prime}(s), \\
a_{2}(t, s)=\frac{1}{2}\left[b_{2}(x(t), x(s))+(\log 2) b_{1}(x(t), x(s))\right] x^{\prime}(s), \quad t, s \in \mathbb{R} .
\end{gathered}
$$

Clearly, $f$ is 1-periodic and even, whereas $a_{1}$ and $a_{2}$ are 1-biperiodic, even in $t$ and odd in $s$. The relation between solutions of (1) and (2) is somewhat more sophisticated: for $s \in\left(-\frac{1}{2}, \frac{1}{2}\right]$

$$
u(s)= \begin{cases}v(x(s)), & 0 \leq s \leq \frac{1}{2}, \\ -v(x(-s)), & -\frac{1}{2}<s<0\end{cases}
$$

and after that $u$ is extended from $\left(-\frac{1}{2}, \frac{1}{2}\right]$ to $\mathbb{R} 1$-periodically. Thus $u$ is a 1-periodic odd function. To the generalized solution $(\omega, v)$ of (1) there corresponds the generalized solution $(\omega, u)$ of (2) satisfying $\omega \varphi+\mathcal{A} u=f$, where $\varphi(t)=$ $\psi(x(t)), t \in \mathbb{R}$.

\section{SOLVABILITY OF THE PROBLEM}

Notice that $a_{1}, a_{2} \in C^{m}(\mathbb{R}), f, \varphi \in C^{m}(\mathbb{R})$ if $b_{1}, b_{2} \in C^{m}([-1,1] \times[-1,1])$, $g, \psi \in C^{m}[-1,1]$. Introduce the Sobolev space $H^{\lambda}, \lambda \geq 0$, of 1 -periodic functions $u$ having a finite norm

$$
\|u\|_{\lambda}=\left(\sum_{k \in \mathbb{Z}} \underline{k}^{2 \lambda}|\hat{u}(k)|^{2}\right)^{1 / 2}, \underline{k}=\max \{1,|k|\}, \hat{u}(k)=\int_{-1 / 2}^{1 / 2} u(s) e^{-i k 2 \pi s} d s .
$$

We have $H^{\lambda}=H_{\mathrm{ev}}^{\lambda} \oplus H_{\mathrm{od}}^{\lambda}$, where $H_{\mathrm{ev}}^{\lambda}$ and $H_{\mathrm{od}}^{\lambda}$ are closed subspaces of $H^{\lambda}$ consisting of even and odd functions, respectively. An orthogonal basis of $H_{\mathrm{ev}}^{\lambda}$ is given by $\{\cos (k 2 \pi t)\}_{k \geq 0}$, and an orthogonal basis of $H_{\mathrm{od}}^{\lambda}$ is given by $\{\sin (k 2 \pi t)\}_{k \geq 1}$. We also introduce the Sobolev space $H^{\lambda_{1}, \lambda_{2}}, \lambda_{1} \geq 0, \lambda_{2} \geq 0$, of 1-biperiodic functions $a$ having a finite norm

$$
\begin{aligned}
\|a\|_{\lambda_{1}, \lambda_{2}} & =\left(\sum_{\left(k_{1}, k_{2}\right) \in \mathbb{Z}^{2}} \underline{k}_{1}^{2 \lambda_{1}} \underline{k}_{1}^{2 \lambda_{2}}\left|\hat{a}\left(k_{1}, k_{2}\right]\right|^{2}\right)^{1 / 2}, \\
\hat{a}\left(k_{1}, k_{2}\right) & =\int_{-1 / 2}^{1 / 2} \int_{-1 / 2}^{1 / 2} a(t, s) e^{-i k_{1} 2 \pi t} e^{-i k_{2} 2 \pi s} d s d t,
\end{aligned}
$$


and the subspace $H_{\mathrm{ev}, \mathrm{od}}^{\lambda_{1}, \lambda_{2}}$ of functions which are even in the first argument and odd in the second argument.

It is well known that

$$
\begin{gathered}
A_{0} \sin (k 2 \pi t)=-\cos (k 2 \pi t), \quad k \geq 1, \\
A_{0} 1=0, \quad A_{0} \cos (k 2 \pi t)=\sin (k 2 \pi t), \quad k \geq 1 .
\end{gathered}
$$

Thus $A_{0} \in \mathcal{L}\left(H_{\text {od }}^{\lambda}, H_{\text {ev }}^{\lambda}\right)$ is a Fredholm operator of index -1 for every $\lambda \geq 0$.

Lemma 2.1. If $a_{1} \in H_{\mathrm{ev}, \mathrm{od}}^{\mu, \nu} \cap H_{\mathrm{ev}, \mathrm{od}}^{\nu,}, \frac{1}{2}<\nu \leq \mu$, then $A_{1} \in \mathcal{L}\left(H_{\mathrm{od}}^{\lambda}, H_{\mathrm{ev}}^{\lambda}\right)$ is compact for every $\lambda \in[0, \mu]$.

Lemma 2.2. If $a_{2} \in H_{\mathrm{ev}, \mathrm{od}}^{\mu, 0}, \mu \geq 0$, then $A_{2} \in \mathcal{L}\left(H_{\mathrm{od}}^{\lambda}, H_{\mathrm{ev}}^{\lambda}\right)$ is compact for every $\lambda \in[0, \mu]$.

Lemma 2.3. Assume that $a_{1} \in H_{\mathrm{ev}, \mathrm{od}}^{\mu, \nu} \cap H_{\mathrm{ev}, \mathrm{od}}^{\nu,}, a_{2} \in H_{\mathrm{ev}, \mathrm{od}}^{\mu, 0}, \frac{1}{2}<\nu \leq \mu$. Then $\mathcal{A}=A_{0}+A_{1}+A_{2} \in \mathcal{L}\left(H_{\mathrm{od}}^{\lambda}, H_{\mathrm{ev}}^{\lambda}\right)$ is a Fredholm operator of index -1 for every $\lambda \in[0, \mu]$.

The proofs of Lemmas 2.1-2.3 can be constructed following the ideas of $\left.{ }^{4}\right]$. As a consequence of Lemma 2.3 we obtain the following result.

Theorem 2.1. Assume the conditions of Lemma 2.3. Assume also that the homogeneous equation $\mathcal{A} u=0$ has in $H_{\mathrm{od}}^{\mu}$ only the trivial solution. Then the range $\mathcal{A} H_{\mathrm{od}}^{\mu} \subset H_{\mathrm{ev}}^{\mu}$ is of codimension 1. Fixing a $\varphi \in H_{\mathrm{ev}}^{\mu} \backslash \mathcal{A} H_{\mathrm{od}}^{\mu}$, for every $f \in H_{\mathrm{ev}}^{\mu}$ we get a unique pair $(\omega, u) \in \mathbb{C} \times H_{\text {od }}^{\mu}$ such that $\omega \varphi+\mathcal{A} u=f$, and this generalized solution of (2) is unique in $\mathbb{C} \times H_{\text {od }}^{0}$.

We have $H^{\mu} \subset C^{m}(\mathbb{R})$ for $m<\mu-\frac{1}{2}, \mu>\frac{1}{2}$, and under conditions of Theorem 2.1, $u \in C^{m}(\mathbb{R})$. For $(\omega, v)$, the generalized solution of (1), we have

$$
v(x)=u\left(\frac{1}{2 \pi} \arccos (-x)\right), \quad 1 \leq x \leq 1 .
$$

So $v$ is continuous on $[-1,1], C^{m}$-smooth in $(-1,1)$, satisfies $v(-1)=u(0)=0$, $v(1)=u(1 / 2)=0$, but the derivatives of $v$ have certain singularities at the end points of the interval $(-1,1)$, e.g. $v \in C^{1}(-1,1)$ for $\mu>\frac{3}{2}$,

$$
v^{\prime}(x)-\frac{u^{\prime}(0)}{2 \pi \sqrt{1-x^{2}}} \rightarrow 0 \text { as } x \rightarrow-1, \quad v^{\prime}(x)-\frac{u^{\prime}\left(\frac{1}{2}\right)}{2 \pi \sqrt{1-x^{2}}} \rightarrow 0 \text { as } x \rightarrow 1
$$




\section{A FULLY DISCRETE COLLOCATION METHOD}

For $N \in \mathbb{N}$, introduce $m, M, n \in \mathbb{N}$ such that

$$
\begin{gathered}
2 m \leq M \leq n \leq N, \quad m \sim N^{\varrho}, \quad M \sim N^{\sigma}, \quad n \sim N^{\tau}, \\
0<\varrho \leq \sigma \leq \tau<1, \quad \sigma \leq \frac{1}{2}, \quad \frac{\mu}{\mu+1} \leq \tau<1,
\end{gathered}
$$

where $n \sim N^{\tau}$ means that there are positive constants $c_{1}$ and $c_{2}$ such that $c_{1} \leq n N^{-\tau} \leq c_{2}$ as $N \rightarrow \infty$. We approximate $\mathcal{A}=A_{0}+A_{1}+A_{2} \in \mathcal{L}\left(H_{\mathrm{od}}^{0}, H_{\mathrm{ev}}^{0}\right)$ by $\mathcal{A}_{N} \in \mathcal{L}\left(H_{\mathrm{od}}^{0}, H_{\mathrm{ev}}^{0}\right)$ defined by

$$
\mathcal{A}_{N}=A_{0}+Q_{M}^{\mathrm{ev}}\left(A_{1}^{(M)}+A_{2}^{(M)}\right) P_{m}^{\mathrm{od}}+Q_{n}^{\mathrm{ev}} A_{1}^{[d]}\left(P_{n}^{\mathrm{od}}-P_{m}^{\mathrm{od}}\right),
$$

where $P_{n}^{\text {od }}$ is the orthogonal projection operator in $H_{\text {od }}^{0}$ to

$$
\mathcal{T}_{n}^{\text {od }}=\operatorname{span}\{\sin (k 2 \pi t), \quad k=1, \ldots, n\} ;
$$

$Q_{n}^{\mathrm{ev}}$ is the interpolation projection operator defined by

$$
\begin{gathered}
Q_{n}^{\mathrm{ev}} u \in \mathcal{T}_{n}^{\mathrm{ev}}=\operatorname{span}\{\cos (k 2 \pi t), k=0,1, \ldots, n\}, \\
\left(Q_{n} u\right)\left(\frac{j}{2 n+1}\right)=u\left(\frac{j}{2 n+1}\right), \quad j=0,1, \ldots, n, \quad u \in H_{\mathrm{ev}}^{\mu}, \quad \mu>\frac{1}{2} ;
\end{gathered}
$$

the product integration approximations $A_{1}^{(M)}, A_{2}^{(M)} \in \mathcal{L}\left(H_{\text {od }}^{\mu}, H_{\text {ev }}^{0}\right)$ are defined by

$$
\begin{aligned}
\left(A_{1}^{(M)} u\right)(t) & =\int_{-1 / 2}^{1 / 2} \log |\sin \pi(t-s)| Q_{M, s}^{\mathrm{ev}}\left(a_{1}(t, s) u(s)\right) d s, \\
\left(A_{2}^{(M)} u\right)(t) & =\int_{-1 / 2}^{1 / 2} Q_{M, s}^{\mathrm{ev}}\left(a_{2}(t, s) u(s)\right) d s, \quad u \in H_{\mathrm{od}}^{\mu}, \quad \mu>\frac{1}{2},
\end{aligned}
$$

where the index $s$ in $Q_{M, s}^{\mathrm{ev}}$ indicates the interpolation with respect to the argument $s$; the asymptotic approximation $A_{1}^{[d]} \in \mathcal{L}\left(H_{\mathrm{od}}^{0}, H_{\mathrm{ev}}^{0}\right)$ of $A_{1}$ is defined by

$$
\begin{array}{r}
A_{1}^{[d]} \sin (k 2 \pi t)=\sum_{j=0}^{d-2} k^{-1-j} b_{j}(t)\left\{\begin{array}{ll}
\sin (k 2 \pi t), & j \text { even } \\
\cos (k 2 \pi t), & j \text { odd }
\end{array}\right\}, \quad k=1,2, \ldots, \\
b_{j}(t)=-\left.\left\{\begin{array}{ll}
(-1)^{j / 2}, & j \text { even } \\
(-1)^{(j-1) / 2}, & j \text { odd }
\end{array}\right\} \frac{1}{2} \frac{1}{(2 \pi)^{j}}\left(\frac{\partial}{\partial s}\right)^{j} a_{1}(t, s)\right|_{s=t}, \\
j=0, \ldots, d-2,
\end{array}
$$


$\mathbb{N} \ni d \geq \frac{1-\varrho}{\varrho} \mu, \quad \mu>\frac{1}{2} ; \quad d=1, \quad A_{1}^{[d]}=0$ may be set if $\frac{1-\varrho}{\varrho} \mu \leq 1$

Lemma 3.1. Let (3) be fulfilled with a $\mu>\frac{1}{2}$, and let $d \geq \frac{1-\varrho}{\varrho} \mu$. Further, assume that $a_{i}=a_{i}(t, s), i=1,2$, are even in $t$, odd in $s$ and with a $\nu>1 / 2$,

$$
\begin{aligned}
& a_{1} \in H^{\nu, d+\nu} \cap H^{\mu+1, \nu} \cap H^{\nu+\mu(1-\sigma) / \sigma, \mu / \sigma} \cap H^{\mu / \sigma, \nu+\mu(1-\sigma) / \sigma}, \\
& a_{2} \in H^{\nu, \mu / \sigma} \cap H^{\mu / \sigma, 0} \cap H^{0, \mu(1-\varrho) / \varrho} .
\end{aligned}
$$

Then

$$
\begin{gathered}
\left\|\mathcal{A}-\mathcal{A}_{N}\right\|_{\lambda, \mu}:=\left\|\mathcal{A}-\mathcal{A}_{N}\right\|_{\mathcal{L}\left(H_{\mathrm{ev}}^{\mu}, H_{\mathrm{od}}^{\lambda}\right)} \leq c N^{\lambda-\mu} \quad(0 \leq \lambda \leq \mu), \\
\left\|\mathcal{A}-\mathcal{A}_{N}\right\|_{\lambda, \lambda} \rightarrow 0 \text { as } N \rightarrow \infty \quad(0 \leq \lambda \leq \mu) .
\end{gathered}
$$

Theorem 3.1. Assume the conditions of Lemma 3.1. Assume also that the homogeneous equation $\mathcal{A} u=0$ has in $H_{\mathrm{od}}^{\mu}$ only the trivial solution. Let $\varphi \in H_{\mathrm{ev}}^{\mu} \backslash \mathcal{A} H_{\mathrm{od}}^{\mu}$. Then there is a $N_{0} \in \mathbb{N}$ such that for $N \geq N_{0}$, the approximate problem

$$
\omega Q_{N}^{\mathrm{ev}} \varphi+\mathcal{A}_{N} u=Q_{N}^{\mathrm{ev}} f
$$

has for every $f \in H_{\mathrm{ev}}^{\mu}$ a solution $\left(\omega_{N}, u_{N}\right) \in \mathbb{C} \times \mathcal{T}_{N}^{\mathrm{od}}$ which is unique in $\mathbb{C} \times H_{\mathrm{od}}^{0}$, and

$$
\left|\omega_{N}-\omega\right| \leq c N^{-\mu}\|f\|_{\mu}, \quad\left\|u_{N}-u\right\|_{\lambda} \leq c N^{\lambda-\mu}\|f\|_{\mu} \quad(0 \leq \lambda \leq \mu),
$$

where $(\omega, u) \in \mathbb{C} \times H_{\mathrm{ev}}^{\mu}$ is the (unique) solution of the problem $\omega \varphi+\mathcal{A} u=f$.

Notice that to $\left(\omega_{N}, u_{N}\right), u_{N}=\sum_{j=1}^{N} c_{j} \sin (j 2 \pi t)$, there corresponds the approximate generalized solution $\left(\omega_{N}, v_{N}\right)$ of (1) with

$$
\begin{aligned}
v_{N}(x)=u_{N}\left(\frac{1}{2 \pi} \arccos (-x)\right) & =\sum_{j=1}^{N} c_{j} \sin (j \arccos (-x)) \\
& =\sqrt{1-x^{2}} \sum_{j=1}^{N} c_{j} U_{j-1}(-x),
\end{aligned}
$$

where $U_{j}(x)=\sin ((j+1) \arccos x) / \sqrt{1-x^{2}}, j=0,1, \ldots$, are the Chebyshev polynomials of the second kind. Moreover, by Theorem 3.1

$$
\left\|v_{N}-v\right\|_{L_{\sigma}^{2}}=\left\|u_{N}-u\right\|_{0} \leq c N^{-\mu}\|f\|_{\mu},
$$

where $(\omega, v), v(x)=u\left(\frac{1}{2 \pi} \arccos (-x)\right)$, is the generalized solution of problem (1). Also estimates of $v_{N}-v$ in weighted Sobolev norms follow from Theorem 3.1. 


\section{MATRIX FORM OF THE METHOD AND CONJUGATE GRADIENTS}

The dimension of the problem (5) can be reduced from $N$ to $n$. Namely, if $\left(\omega_{N}, u_{N}\right)$ with $u_{N}=\sum_{j=1}^{N} c_{j} \sin (j 2 \pi t)$ is the solution of (5), then $\omega_{n}=\omega_{N}$, $u_{n}=P_{n}^{\text {od }} u_{N}=\sum_{j=1}^{n} c_{j} \sin (j 2 \pi t)$ is the solution of the problem

$$
\omega \varphi_{n}+\mathcal{A}_{N} u=f_{n}
$$

with $\varphi_{n}=P_{n}^{\mathrm{ev}} Q_{N}^{\mathrm{ev}} \varphi, f_{n}=P_{n}^{\mathrm{ev}} Q_{N}^{\mathrm{ev}} f$, and $u_{N}$ can be reconstructed by the formula $u_{N}=u_{n}+\sum_{j=n+1}^{N}\left(\omega_{n} \alpha_{j}-d_{j}\right) \sin (j 2 \pi t)$, where $\alpha_{j}$ and $d_{j}$ are the Fourier coefficients of $Q_{N}^{\mathrm{ev}} \varphi$ and $Q_{N}^{\mathrm{ev}} f$, respectively,

$$
Q_{N}^{\mathrm{ev}} \varphi=\sum_{j=0}^{N} \alpha_{j} \cos (j 2 \pi t), \quad Q_{N}^{\mathrm{ev}} f=\sum_{j=0}^{N} d_{j} \cos (j 2 \pi t) .
$$

Denoting $\underline{c}_{n}=\left(c_{1}, \ldots, c_{n}\right)^{\top}, \underline{d}_{n}=\left(d_{0}, d_{1}, \ldots, d_{n}\right)^{\top}, \underline{\alpha}_{n}=\left(\alpha_{0}, \alpha_{1}, \ldots, \alpha_{n}\right)^{\top}$, we have problem (6) in the matrix form

$$
\omega \underline{\alpha}_{n}+\mathbb{M}_{n} \underline{c}_{n}=\underline{d}_{n}
$$

with the $(n+1) \times n$ matrix $\mathbb{M}_{n}$ defined by

$$
\begin{aligned}
\mathbb{M}_{n}=\mathbb{A}_{0} & +\mathbb{I}_{n, M} \tilde{\mathcal{C}}_{M}\left(\mathbb{A}_{1}^{(M)}+\mathbb{A}_{2}^{(M)}\right) \mathcal{S}_{M} \mathbb{P}_{M, m, n} \\
& +\tilde{\mathcal{C}_{n}} \sum_{j=0}^{d-2} \mathbb{B}_{n}^{(j)}\left\{\begin{array}{ll}
\mathcal{C}_{n} \mathbb{J}_{n}, & j \text { even } \\
\mathbb{J}_{n} \mathcal{S}_{n}, & j \text { odd }
\end{array}\right\} \mathbb{G}_{N}^{(j)},
\end{aligned}
$$

where

$$
\mathbb{A}_{0}=-\mathbb{J}_{n}, \mathbb{J}_{n}=\left(\begin{array}{c}
0 \\
\mathbb{I}_{n}
\end{array}\right) \text { are }(n+1) \times n \text { matrices, }
$$

$\mathbb{I}_{n}$ is an $n \times n$ identity matrix,

$$
\begin{aligned}
& \mathbb{I}_{n, M}=\left(\begin{array}{c}
\mathbb{I}_{M+1} \\
0
\end{array}\right) \text { is an }(n+1) \times(M+1) \text { matrix, } \\
& \mathbb{P}_{M, m, n}=\left(\begin{array}{cc}
\mathbb{I}_{m} & 0 \\
0 & 0
\end{array}\right) \text { is an } M \times n \text { matrix; } \\
& \mathcal{C}_{n}=\left(\cos \left(k j \frac{2 \pi}{2 n+1}\right)\right)_{j, k=0}^{n}, \tilde{\mathcal{C}}_{n}=\frac{4}{2 n+1} \mathbb{D}_{n} \mathcal{C}_{n} \mathbb{D}_{n}, \\
& \mathbb{D}_{n}=\operatorname{diag}\left\{\frac{1}{2}, 1, \ldots, 1\right\}, \mathcal{S}_{n}=\left(\sin \left(k j \frac{2 \pi}{2 n+1}\right)\right)_{j, k=1}^{n} ; \\
& \mathbb{A}_{1}^{(M)}=\left(a_{k j}^{(1)}\right), \mathbb{A}_{2}^{(M)}=\left(a_{k j}^{(2)}\right) \text { are }(M+1) \times M \text { matrices with the entries }
\end{aligned}
$$




$$
\begin{aligned}
& a_{k j}^{(1)}=-\frac{1}{2 M+1} a_{1}\left(\frac{k}{2 M+1}, \frac{j}{2 M+1}\right)\left(\gamma_{|k-j|}+\gamma_{k+j}\right), \\
& a_{k j}^{(2)}=\frac{2}{2 M+1} a_{2}\left(\frac{k}{2 M+1}, \frac{j}{2 M+1}\right), \quad k=0,1, \ldots, M, \quad j=1, \ldots, M, \\
& \gamma_{k}=\log 2+\sum_{l=1}^{M} \frac{1}{l} \cos \left(k l \frac{2 \pi}{2 M+1}\right), \quad k=0,1, \ldots, M, \\
& \gamma_{M+k}=\gamma_{M+1-k}, \quad 1 \leq k \leq M ; \\
& \mathbb{G}_{n}^{(j)}=\operatorname{diag}\left\{0, \ldots, 0,(m+1)^{-1-j}, \ldots, n^{-1-j}\right\} \text { is an } n \times n \text { matrix, } \\
& \mathbb{B}_{n}^{(j)}=\operatorname{diag}\left\{b_{j}(0), b_{j}\left(\frac{1}{2 n+1}\right), \ldots, b_{j}\left(\frac{n}{2 n+1}\right)\right\} \text { is an }(n+1) \times(n+1)
\end{aligned}
$$

matrix.

The application of $\mathbb{M}_{n}$ to an $n$-vector, as well as the application of $\mathbb{M}_{n}^{\prime}$, the Hermite adjoint matrix of $\mathbb{M}_{n}$, to an $(n+1)$-vector costs $\mathcal{O}(n \log n)+\mathcal{O}\left(M^{2}\right)=$ $\mathcal{O}\left(N^{\tau} \log N\right)+\mathcal{O}\left(N^{2 \sigma}\right)$ arithmetical operations, provided that the fast Fourier technique is used for the cosine and sine transformations $\mathcal{C}_{n}$ and $\mathcal{S}_{n}$. The computation of the entries of $\mathbb{M}_{n}$ costs $\mathcal{O}\left(M^{2}\right)+\mathcal{O}(N)=\mathcal{O}(N)$ arithmetical operations. This enables us to design fast solvers of problem (2) on the basis of iteration methods. We specify a classical conjugate gradient iteration algorithm (see $\left[{ }^{6,7}\right]$ ) to solve (7).

Denote by $\underline{x}_{n}=\left(\omega, c_{1}, \ldots, c_{n}\right)$ the $(n+1)$-vector of unknowns and rewrite the system (7) in the form

$$
\mathbb{A}_{n} \underline{x}_{n}=\underline{d}_{n},
$$

where

$$
\mathbb{A}_{n}=\left(\begin{array}{ll}
\underline{\alpha}_{n} & \mathbb{M}_{n}
\end{array}\right) \text { is an }(n+1) \times(n+1) \text { matrix } .
$$

\section{Algorithm 1.}

Step 0: $\underline{x}_{n}^{0}=0, \underline{y}_{n}^{0}=-\underline{d}_{n}, \underline{r}_{n}^{0}=-\mathbb{A}_{n}^{\prime} \underline{d}_{n}$.

For $k=0,1,2 \ldots$ :

(i) if $\left\|\underline{y}_{n}^{k}\right\| \leq\left\|\underline{d}_{n}\right\| \delta N^{-\mu}$, then terminate;

(ii) if $\left\|\underline{y}_{n}^{k}\right\|>\left\|\underline{d}_{n}\right\| \delta N^{-\mu}$, then go to step $k+1$, and compute

$$
\begin{aligned}
\underline{z}_{n}^{k} & = \begin{cases}-\underline{r}_{n}^{0}, & k=0, \\
-\underline{r}_{n}^{k}+\left(\left\|\underline{r}_{n}^{k}\right\| /\left\|\underline{r}_{n}^{k-1}\right\|\right)^{2} \underline{z}_{n}^{k-1}, & k \geq 1,\end{cases} \\
\underline{x}_{n}^{k+1} & =\underline{x}_{n}^{k}+\gamma_{k} \underline{z}_{n}^{k}, \quad \gamma_{k}=\left(\left\|\underline{r}_{n}^{k}\right\| /\left\|\mathbb{A}_{n} \underline{z}_{n}^{k}\right\|\right)^{2}, \\
\underline{y}_{n}^{k+1} & =\underline{y}_{n}^{k}+\gamma_{k} \mathbb{A}_{n} \underline{z}_{n}^{k}, \\
\underline{r}_{n}^{k+1} & =\underline{r}_{n}^{k}+\gamma_{k} \mathbb{A}_{n}^{\prime} \mathbb{A}_{n} \underline{z}_{n}^{k} .
\end{aligned}
$$


In this algorithm the usual norm $\left\|\underline{d}_{n}\right\|=\left(\sum_{k=0}^{n}\left|d_{k}\right|^{2}\right)^{1 / 2}$ is used for $(n+1)$ vectors. We have incorporated the residual termination rule into the algorithm: the iterations stop on the first $k$ such that $\left\|\mathbb{A}_{n} \underline{x}_{n}^{k}-\underline{d}_{n}\right\| \leq\left\|\underline{d}_{n}\right\| \delta N^{-\mu}$. Here $\delta>0$ is a parameter.

Theorem 4.1. Under conditions of Theorem 3.1, for $N \geq N_{0}$, Algorithm 1 terminates at an iteration number $k$ of order $o(\log N)$ as $N \rightarrow \infty$. The corresponding iteration approximation $\underline{x}_{n}^{k}=\left(\omega^{k}, c_{1}^{k}, \ldots, c_{n}^{k}\right)$ defines an iteration solution $\left(\omega_{N}^{k}, u_{N}^{k}\right)$ to (5) with

$$
\omega_{N}^{k}=\omega^{k}, \quad u_{N}^{k}=\sum_{j=1}^{n} c_{j}^{k} \sin (j 2 \pi t)+\sum_{j=n+1}^{N}\left(\omega^{k} \alpha_{j}-d_{j}\right) \sin (j 2 \pi t)
$$

for which there hold the optimal order estimates

$$
\left|\omega_{N}^{k}-\omega\right| \leq c N^{-\mu}\|f\|_{\mu}, \quad\left\|u_{N}^{k}-u\right\|_{\lambda} \leq c N^{\lambda-\mu}\|f\|_{\mu}, \quad 0 \leq \lambda \leq \mu,
$$

where $(\omega, u) \in \mathbb{C} \times H_{\text {od }}^{\mu}$ is the unique generalized solution of integral equation (2).

The computation of $\underline{d}_{N}=\tilde{\mathcal{C}}_{N} \underline{f}_{N}$ and $\underline{\alpha}_{N}=\tilde{\mathcal{C}}_{N} \underline{\varphi}_{N}$ from the vectors of grid values $\underline{f}_{N}=\left(f(0), f\left(\frac{1}{2 N+1}\right), \ldots, f\left(\frac{N}{2 N+1}\right)\right)$ and $\underline{\varphi}_{N}=\left(\varphi(0), \varphi\left(\frac{1}{2 N+1}\right)\right.$, $\left.\ldots, \varphi\left(\frac{N}{2 N+1}\right)\right)$ by the fast algorithm $\operatorname{costs} \mathcal{O}(N \log N)$ arithmetical operations. All other computations are cheaper, costing asymptotically $o(\log N)\left(\mathcal{O}\left(N^{\tau} \log N\right)+\right.$ $\mathcal{O}\left(N^{2 \sigma}\right)$ ) arithmetical operations, which is $o(N)$ for $\sigma<\frac{1}{2}$; notice that an iteration step by Algorithm 1 contains one application of $\mathbb{A}_{n}$ and one application of $\mathbb{A}_{n}^{\prime}$. If the Fourier coefficients of $f$ and $\varphi$ with respect to $\cos (k 2 \pi t)(k=0,1,2, \ldots)$ are known, we can use $P_{N}^{\mathrm{ev}} f$ and $P_{N}^{\mathrm{ev}} \varphi$ instead of $Q_{N}^{\mathrm{ev}} f$ and $Q_{N}^{\mathrm{ev}} \varphi$. In this case the full number of arithmetical and logical operations reduces to $N+o(N)$.

If functions $a_{1}, a_{2}$, and $\varphi$ are real, then $\mathbb{A}_{n}$ is real.

\section{REFERENCES}

1. Okada, S. and Prössdorf, S. On the solution of the generalized airfoil equation. J. Integral Equations Appl., 1997, 9, 71-98.

2. Berthold, D., Hoppe, W. and Silbermann, B. S. A fast algorithm for solving the generalized airfoil equation. J. Comput. Appl. Math., 1992, 43, 185-219.

3. Saranen, J. and Vainikko, G. Fast collocation solvers for integral equations on open arcs. J. Integral Equations Appl., 1999, 11, 57-102.

4. Vainikko, G. Fast solvers of generalized airfoil equation of index 1. Oper. Theory $A d v$. Appl., 2001, 121, 498-516.

5. Lifanov, I. Singular solutions of singular integral equations and flow ejecting for an arbitrary contour. Sov. J. Numer. Anal. Math. Modelling, 1989, 4, 239-252.

6. Golub, G. H. and van Loan, C. F. Matrix Computations, 2nd ed. The John Hopkins Univ. Pr., Baltimore, 1989.

7. Plato, R. and Vainikko, G. On the fast and fully discretized solution of integral and pseudodifferential equations on smooth curves. Calcolo, 2001, 38, 25-48. 


\section{ÜLDISTATUD TIIVAVÕRRANDI KIIRED LAHENDUSMEETODID INDEKSI -1 KORRAL}

\section{Gennadi VAINIKKO}

Üldistatud tiivavõrrandit on käsitletud situatsioonis, kui vastava integraaloperaatori Fredholmi indeks on -1 . On esitatud vastava laiendatud ülesande lahendusmeetod, mis põhineb trigonomeetrilisele kollokatsioonimeetodile, on aga täielikult diskreetne ning võimaldab teatud mõttes optimaalse täpsusastmega lähislahendi $N$ parameetrit määrata $\mathcal{O}(N \log N)$ aritmeetilise tehtega. 